\title{
Helmintos parásitos de Telmatobius jelskii (Peters) (Anura, Leptodactylidae) de Lima, Perú
}

\author{
José lannacone ${ }^{1}$ \\ Laboratorio de Ecofisiología, Área de Biodiversidad Animal, Facultad de Ciencias Naturales y Matemáticas, Universidad \\ Nacional Federico Villarreal. Calle San Marcos 383, Lima 21, Perú. E-mail: joselorena@terra.com
}

\begin{abstract}
Helminth parasites of Telmatobius jelskii (Peters) (Anura, Leptodactylidae) from Lima, Peru. A quantitative research of parasites of 67 endemic frog Telmatobius jelskii (Peters, 1863) collected from Laguna Tucto (76 $\left.46^{\prime} 11^{\prime \prime} \mathrm{W}, 10^{\circ} 39^{\prime} 11^{\prime \prime S}\right)$ where Pativilca River is originated was conducted, and was located in the Province of Oyon, high Andean area from the Department of Lima, Peru during September-October 2000. Of the frogs collected, 23 were females and 44 males. Male showed a length between $5.2 \pm 0.5 \mathrm{~cm}$ (range $=4.0-6.4 \mathrm{~cm}$ ) and female between $5.5 \pm 1 \mathrm{~cm}$ (range $=3.9-7.6 \mathrm{~cm}$ ) and were not found differences between both sexes. 86 specimens of parasite and three species in total during all the survey were collected. 28 hosts were infected (41.8\%). twenty-five hosts (37.3\%) showed infection with one parasite species, and three (4.5\%) had two parasite species. Three parasite species were found: Gorgoderina parvicava Travassos, 1922 (Digenea: Gorgoderidae) (Prevalence $=40.3 \%$; mean Intensity $=3.1$; mean abundance $=1.2$ ), Cylindrotaenia americana Jewell, 1916 (Cestoda: Proteocephalidae) (Prevalence $=3 \%$; mean Intensity $=1$; mean abundance $=0.02$ ) and Aplectana hylambatis (Baylis, 1927) (Nematoda: Cosmocercidae) (Prevalence $=3 \%$; mean Intensity $=1$; mean abundance $=0.02$ ). $G$. parvicava had an overdispersed distribution and was the dominant species. An effect of sex and length with prevalence and mean abundance of infection of $G$. parvicava was not found. The relationship of helminthes parasites with $T$. jelskii is discussed. G. parvicava and $C$. americana are new records for $T$. jelskii.
\end{abstract}

KEY WORDS. Aplectana, Cylindrotaenia, Gorgoderina, parasite ecology.

La disminución en las poblaciones de anfibios en diversas regiones del mundo, así como la aparición de malformaciones en algunos miembros de aquéllas, han llamado la atención hacia este grupo para ser considerados como organismos "centinelas" en la detección de alteraciones ambientales, por lo que son motivo de intensos monitoreos en diversas partes del mundo. Además, los endoparásitos de anfibios proporcionan importante información en estudios evolutivos, biogeográficos, como indicadores ecológicos de hábitat y de comportamiento preferencial reproductivo y alimentario de sus hospederos anuros (Esch \& Fernández 1993, BRooks et al. 2001).

El Perú con 315 especies registradas de anfibios, se encuentra en cuarto lugar a nivel mundial, después de tres países Neotropicales como Brasil, Colombia y Ecuador (Rodríguez et al. 1993). Telmatobius jelskii (Peters, 1863) (Leptodactylidae), es una anfibio que presenta una distribución geográfica conocida que abarca Ayacucho, Huancavelica, Junín y Lima. En la zona de Oyón, Lima, Perú, T. jelskii "chegiaj" es usado en la etnomedicina como desinflamante y como alimento (RodríGUEZ et al. 1993, Frost 1985).

Con relación a la fauna parasitaria de $T$. jelskii, se han registrado a la fecha cuatro nematodos parásitos: Aplectana hylambatis
(Baylis, 1927), Camallanus sp., Falcaustra condorcanqui Ibáñez \& Córdova, 1976 y Hedruris sp. (TANTALEÁn et al. 1992, Sarmiento et al. 1999). Además, la mayoría de los trabajos realizados hasta la fecha en fauna parasitaria de anfibios, son de índole cualitativa y de amplitud de ámbito (TANTALEÄN \& GarCía 1989, Tantalé́n et al. 1992, Recharte 1995, Sarmiento et al. 1999) y no se ha efectuado ningún análisis cuantitativo de las comunidades parasitarias en este anuro.

Este trabajo representa un análisis cuantitativo de las comunidades parasitarias de T. jelskii con el objetivo de evaluar la influencia del tamaño y sexo del hospedero, sobre las especies componentes de su fauna parasitaria.

\section{MATERIAL Y MÉTODOS}

Se colectaron 67 especimenes de T. jelskii de distribución restringida al Perú, entre septiembre y octubre del 2000 en la desembocadura de la Laguna de Tucto a $4200 \mathrm{msnm}\left(76^{\circ} 46^{\prime}\right.$ $\left.11^{\prime \prime} \mathrm{W}, 10^{\circ} 39^{\prime} 11^{\prime \prime} \mathrm{S}\right)$ que da origen al río Pativilca, ubicado en la Provincia de Oyón, zona altoandina del Departamento de Lima. Desde el punto de vista geográfico, la zona pertenece al Dominio Andino-patagónico y a la Ecorregión Puna (CABRERA 
\& W ILLINK 1980). Los anfibios fueron colectados en horas diurnas utilizando redes de pesca artesanal. Cada espécimen se llevó al laboratorio en forma individual en bolsas plásticas de $10 \times 12 \mathrm{~cm}$ tratando que se mantuvieran en óptimo estado de conservación, siendo sacrificados mediante inmersión en agua tibia a $40^{\circ} \mathrm{C}$ y posteriormente fueron identificados siguiendo los criterios de Frost (1985). Los parásitos se colectaron, fijaron, preservaron, colorearon y montaron siguiendo las recomendaciones de Eiras et al. (2000). Para la búsqueda de metazoos parásitos se incluyó intestino delgado y grueso, hígado, pulmón y vejiga urinaria. Para la determinación taxonómica de los parásitos se usaron los criterios taxonómicos dados por ANDERSON (1992) y Khall et al. (1994) Especimenes representativos de las especies colectadas fueron depositados en la Colección Helmintológica del Museo de Historia Natural de la Universidad Nacional Mayor de San Marcos (MUSM-UNMSM). Gorgoderina parvicava Travassos, 1922 MUSM no. 1762 y Aplectana hylambatis (Baylis, 1927) MUSM no. 1763. Especimenes representativos de C. americana fueron depositados en la helmintoteca de la Facultad de Ciencias Naturales y Matemáticas de la Universidad Nacional Federico Villarreal.

Se determinó en los hospederos el sexo, examinado la cavidad corporal y específicamente las gónadas, y la longitud del hocico a la cloaca $(\mathrm{cm})$. La longitud de los hospederos se dividió en seis rangos de aproximadamente 0,5 cada uno. Estos rangos fueron: $3,9-4,4(\mathrm{n}=5) ; 4,5-4,9(\mathrm{n}=16) ; 5,0-5,4(\mathrm{n}=18)$; $5,5-5,9(\mathrm{n}=19) ; 6,0-6,4(\mathrm{n}=4) ; 6,5-6,9(\mathrm{n}=2)$ y 7,0-7,6 $(\mathrm{n}=3)$.

De los anuros colectados fueron 44 machos y 23 hembras. Los machos mostraron una longitud de $5,2 \pm 0,5 \mathrm{~cm}$ (rango = $4,0-6,4 \mathrm{~cm})$ y las hembras de $5,5 \pm 1 \mathrm{~cm}(\operatorname{rango}=3,9-7,6 \mathrm{~cm})$ no encontrándose diferencias entre ambos sexos. Además, los machos mostraron un peso de $12,4 \pm 2,1 \mathrm{~g}$ (rango $=8,5-18,1 \mathrm{~g}$ ) y las hembras de $12,3 \pm 2,4 \mathrm{~g}$ (rango $=9,8-19,5 \mathrm{~g}$ ) no encontrándose diferencias entre ambos sexos.

Se determinó la prevalencia, intensidad media y abundancia media para todos los parásitos encontrados (BusH et al. 1997). El coeficiente de dispersión (CD) empleado, se determinó de la relación entre varianza $\left(\mathrm{S}^{2}\right)$ /intensidad media. La aproximación ecológica de los metazoos parásitos a nivel de sus comunidades se hizo solo para las especies con prevalencias mayores al 10\%, de acuerdo a Esch et al. (1990).

La prueba de t de Student, previa evaluación de homogeneidad de varianzas empleando la prueba de Levene, fue usada para determinar si la longitud de las ranas hospederos machos y hembras presentaban diferencias significativas. La influencia del tamaño del hospedero en la prevalencia de los parásitos se determinó usando el coeficiente de correlación de Spearman (r). Este se utilizó nuevamente para determinar la relación del tamaño del hospedero con la riqueza específica y para cada parásito. En adición, este coeficiente permitió determinar la relación lineal existente entre la riqueza parasitaria específica y la abundancia total. Se aplicó la prueba $X^{2}$ para tablas de contingencia para determinar el grado de dependencia entre el sexo del hospedero y la prevalencia parasitaria. El posible efecto del sexo en la abundancia media parasitaria se calculó utilizando la prueba de t de Student (ZAR 1996).

La terminología ecológica para la prevalencia, abundancia media e intensidad media de infección siguió los criterios de Bush et al. (1997).

\section{RESULTADOS}

En el presente estudio se encontró tres especies de parásitos: G. parvicava (vejiga urinaria), C. americana (intestino delgado) y A. hylambatis (intestino grueso). Se indica la prevalencia, intensidad media y abundancia media de infestación para los tres parásitos encontrados en los 67 hospederos muestreados de T. jelskii (Tab. I). Además en el parásito más prevalente como G. parvicava, se observa una distribución sobredispersa o contagiosa, pues el coeficiente de dispersión fue mayor a 1. La mayor frecuencia de dominancia fue para $G$. parvicava $(95,4 \%)$, luego Cylindrotaenia americana Jewell, 1916 (2,3\%) y A. hylambatis (2,3\%).

Tabla I. Prevalencia, intensidad media y abundancia media de infección de los helmintos parásitos de Telmatobius jelskii de Lima, Perú.

\begin{tabular}{lcccc}
\hline Parásito & $\begin{array}{c}\text { Prevalencia } \\
(\%)\end{array}$ & $\begin{array}{c}\text { Intensidad } \\
\text { media } \\
\text { (Rango) }\end{array}$ & $\begin{array}{c}\text { Abundancia } \\
\text { media }\end{array}$ & $\begin{array}{c}\text { Coeficiente } \\
\text { de } \\
\text { dispersión }\end{array}$ \\
\hline $\begin{array}{l}\text { Digenea, Gorgoderidae } \\
\text { Gorgoderina parvicava }\end{array}$ & 40,3 & $3,1 \pm 2(1-10)$ & $1,20 \pm 1,9$ & 1,3 \\
Cestoda, Proteocephalidae & & & & 0 \\
Cylindrotaenia americana & 3,0 & $1,0 \pm 0(1)$ & $0,02 \pm 0,1$ & 0 \\
$\begin{array}{l}\text { Nematoda, Cosmocercidae } \\
\text { Aplectana hylambatis }\end{array}$ & 3,0 & $1,0 \pm 0(1)$ & $0,02 \pm 0,1$ & 0 \\
\hline
\end{tabular}

El promedio de la longitud de los anuros machos y de las hembras asumiendo igualdad de varianza no fue significativamente diferente $(t=1,48 ; P=0,14)$. Tampoco, el promedio del peso de los anuros machos y de las hembras asumiendo igualdad de varianza fue significativamente diferente $(t=1,67 ; P=0,09)$.

Se observó ausencia de relación lineal entre la talla del hospedero, y la prevalencia, intensidad media y abundancia media de G. parvicava (prevalencia: $\mathrm{r}=-0,20, \mathrm{p}=0,67$; intensidad media de infección: $r=-0,04, p=0,84$; abundancia media: $r=$ $-0,20, p=0,10)$. Tampoco se encontró efecto entre el sexo, y la prevalencia, intensidad media y abundancia media de $G$. parvicava (prevalencia: $X^{2}=0,01, \mathrm{p}=0,90$; intensidad media: $\mathrm{t}=0,98, \mathrm{p}=0,33$; abundancia media: $\mathrm{t}=0,81, \mathrm{p}=0,42$ ).

Veintiocho $(41,8 \%)$ ranas estuvieron parasitadas por lo menos con una especie de parásito. Un total de 86 parásitos fueron colectados, con una abundancia media total de 1,3 \pm 1,9 (1-11). 13 hospederos mostraron de tres a más especimenes por anuro. La riqueza promedio de las especies de parásitos $0,46 \pm 0,58(1-2)$ no estuvo relacionada linealmente con la longitud corporal $(r=-0,21 ; p=0,08)$. La abundancia media total y la riqueza de especies estuvieron relacionadas linealmente $(r=0,95 ; P=0,00)$. El monoparasitismo se encontró en 25 hospederos (37,3\%: G. parvicava con $35,8 \%(\mathrm{n}=24)$ y $C$. americana con $1,5 \%(\mathrm{n}=1)$ ), y el biparasitismo en três hospederos (4,5\%: G. parvicava y A. hylambatis con $3 \%(\mathrm{n}=2)$ y $G$. parvicava y C. americana con $1,5 \%(\mathrm{n}=1))$. 


\section{DISCUSIÓN}

BARTON \& Richard (1996) y Barton (1999) señalan que la composición de la fauna parasitaria de anfibios tropicales ocurre en bajas densidades, indicando que es una fauna parasitaria depauperada.

En el Perú, especies del género Gorgoderina han sido registradas en seis especies y en dos familias diferentes de anfibios: Batrachophrynus macrostomus Peters, 1863, Bufo limensis Werner, 1901, B. spinolosus Wiegmann, 1835, Leptodactylus rhodonotus (Günther, 1868), Telmatobius culeus (Garman, 1875) y T. peruvianus Wiegmann, 1835 (TANTALEAN et al. 1992, TANTALEÁN \& GarCía 1993, IbáÑez 1998). СRibb et al. (2002) señalan que los digeneos gorgoderidos requieren de un hospedero intermediario del grupo de los bivalvos para llegar a su hospedero definitivo. Dentro de los patrones de comportamiento aún desconocidos para $T$. jelskii, aparentemente existe la presencia constante de determinados items alimentos durante su desarrollo ontogénico, entre los cuales se encontrarían los hospederos intermediarios bivalvos del digeneo $G$. parvicava. Esto explicaría la falta de correlación de la prevalencia y la abundancia de este parásito con la longitud del anfibio. Estos patrones se han observado en otros anuros (BAKER 1984). Sin embargo, se requeriría un mayor estudio de la biología del digeneo G. parvicava para poder explicar los resultados obtenidos. G. parvicava es un nuevo parásito para T. jelksii.

Aplectana hylambatis, es una especie de amplia distribución que ha sido registrada en diez especies y en tres familias de anfibios en Perú: Bufo arequipensis Vellard, 1959, B. flavolineatus Vellard, 1959, B. marinus (Linnaeus, 1758), B. spinolosus, B. trifolium Tschudi, 1845, Gastrotheca marsupiata (Duméril \& Bibron, 1841), L. rhodonotus, Pleurodema marmorata (Duméril \& Bibron, 1841), T. jelskii y T. marmoratus (Duméril \& Bibron, 1841) (SARMiento et al. 1999). ANDERSON (1992) indica que Aplectana tiene un ciclo biológico en el que la larva no penetra por la piel al anuro hospedero, sino que la larva es ingerida por los renacuajos y desarrolla en el interior de los mismos, permaneciendo hasta la fase adulta de los anuros. Además puede llegar a los adultos, si es que estos últimos ingieren a los renacuajos portadores de este nemátode.

Cylindrotaenia americana es un cestodo que se le ha reportado en una amplia variedad de anuros norteamericanos y sudamericanos (Arthroleptis, Acris, Bufo, Hyla, Leptodactylus, Pseudacris, Rana, Scaphiopus, Plethodon) (Rego 1995, Goldberg et al. 2002), y ha sido registrada en dos especies y en dos familias diferentes de anfibios en Perú: B. spinolosus y T. marmoratus (Tantaleän \& García 1989, Recharte 1995). Esta especie es reportada como un nuevo parásito para T. jelskii.

Se detectó que la fauna parasitaria de $T$. jelskii presentó carencia de correlación entre la prevalencia y abundancia media con el tamaño y sexo de esta rana, en comparación con otros anuros (BAKER 1984, Goldberg et al. 1998, Gillilland \& MuZzall 1999, Muzzall et al. 2001).

BAKER (1984) y RECHARTE (1995) señalaron que la abundancia y la riqueza de las especies de parásitos están significativamente correlacionadas con la longitud del hospedero. En $T$. jelskii no se encontró ninguna relación entre la longitud del hospedero y la riqueza de especies parásitas. Estos resultados sugieren la ausencia de modificaciones en las relaciones ecológicas en los adultos de este hospedador (MARGOGLIESE 2001).
Aunque, la biología de T. jelskii sea poco conocida, es probable que este patrón aplique a este anuro.

GilliLland \& Muzzall (1999), indicaron que la preferencia a uno de los sexos de los hospederos pudiera ser atribuida a las diferencias en las relaciones ecológicas (comportamiento, hábitat y dieta) de los peces machos y hembras. Nuestros resultados muestran ausencia de efecto del sexo del hospedero sobre la prevalencia, intensidad media y abundancia media parasitaria. En el presente trabajo se repite el mismo patrón encontrado en anuros, donde la mayoría de las especies no mostraron diferencias en la prevalencia y abundancia media parasitaria con relación al sexo del hospedero (YoDER \& CogGINS 1996, McAlpine 1997, Goldberg et al. 2002).

\section{AGRADECIMIENTOS}

El autor expresa su agradecimiento a Richard Huaraz, José Soto y Rosario Reyes por su apoyo en la colecta de los anfibios. A Helena Sisniegas (Consejo Nacional de Ciencia y Tecnología, Lima, Perú) por la determinación de la especie de anuro.

\section{REFERENCIAS BIBLIOGRÁFICAS}

ANDERSON, R.C. 1992. Nematode parasites of vertebrates: their development and transmission. Cambridge, $\mathrm{CAB}$ International, 650p.

BAKER, M.R. 1984. Nematode parasitism in amphibian and reptiles. Canadian Journal of Zoology, Vancouver, 62: 747757.

BARTON, D.P. 1999. Ecology of helminth communities in tropical Australian amphibians. International Journal of Parasitology, New York, 29: 921-926.

Barton, D.P. \& S.J. RichaRds. 1996. Helminth infracommunities in Litoria geniculata (Amphibia: Anura) from Birthday Creek, an upland rainforest stream in northern Queensland, Australia. International Journal of Parasitology, New York, 26: 1381-1385.

Brooks, D.R.; V. León-Regagnon \& G. Perez-Ponce. 2001. Los parásitos y la biodiversidad, p. 245-289. In: H.M. HeRNÁNDEZ; A.N. García; F. Álvarez \& M. Ulloa (Eds.). Enfoques contemporáneos para el estudio de la biodiversidad. México,Instituto de Biología, UNAM, 413p.

Bush, A.O.; K.D. Lafferty; J.L. Lotz \& A.W. Shostak. 1997. Parasitology meets ecology on its own terms: Margolis et al. revisited. Journal of Parasitology, Washington, D.C., 83: 575-583.

Cabrera, A.L.. \& A. Willink. 1980. Biogeografía de América Latina. Washington, D.C., Monografía no 13 , OEA, $2^{\text {nd }}$ ed., $122 \mathrm{p}$.

Cribb, T.H.; L.A. Chisholm \& R.A. Bray. 2002. Diversity in the Monogenean and Digenea: does lifestyle matter. International Journal of Parasitology, New York, 32: 321328.

Eiras, J.; R. Takemoto \& G.C. Pavanelli. 2000. Métodos de estudo e técnicas laboratoriais em parasitología de peixes. Maringá, Editora da Universidade Estadual de Maringá, $171 \mathrm{p}$.

Esch, G.W.; A.W. Shostak; \& T.M. Goater. 1990. Patterns and process in helminth parasite communities: an overview, $\mathrm{p}$. 
1-19. In: G. Еsch; A.C. Bush \& J. Ано (Eds.). Parasite Communities: Patterns and processes. New York, Chapman and Hall, 251p.

Esch, G.W. \& J.C. Fernández. 1993. A functional biology of parasitism: Ecological and evolutionary implications. Cambridge, Chapman \& Hall, 337p.

Frost, D. 1985. Amphibian species of the World. Lawrence, Allen Press, 732p.

Gillilland, M.G. \& P.M. Muzzall. 1999. Helminths infecting froglets of the northern leopard frog (Rana pipiens) from Foggy Botton Marsh, Michigan. Journal of the Helminthological Society of Washington 66: 73-77.

Goldberg, S.R.; C.R. Bursey \& H. KaIser. 1998. Gastrointestinal helminthes of the species of Eleutherodactylus (Anura: Leptodactylidae) from West Indies. Caribbean Journal of Science, Mayagüez, 34: 146-149.

Goldberg, S.R.; C.R. Bursey; J.D. Trujillo \& H. KaISER. 2002. Intestinal helminths of seven frog species from Trinidad and Tobago. Caribbean Journal of Science, Mayagüez, 38: 147150.

IвAÑEZ, N.H. 1998. Mención de algunos trematodos en la fauna helmintológica peruana. Revista Peruana de Parasitologia, Lima, 13: 90-97.

Khalil, L.F.; A. Jones \& R.A. Bray. 1994. Keys to the cestode parasites of vertebrates. Wallingford, Oxon, CAB International, XIII+751p.

Marcogliese, D.J. 2001. Pursuing parasites up the food chain: implications of food web structure and function on parasite communities in aquatic systems. Acta Parasitologica, Warszawa, 46: 82-93.

McAlpine, D.F. 1997. Helminth communities in bullfrogs (Rana catesbeiana), green frogs (Rana clamitans), and leopard frogs (Rana pipiens) from New Brunswick, Canada. Canadian
Journal of Zoology, Vancouver, 75: 1883-1890.

Muzzall, P.M.; M.G. Gillilland; C.S. Summer \& C.J. Mehne. 2001. Helminths communities of green frogs Rana clamitans Latreille, from southwestern Michigan. Journal of Parasitology, Lawrence, 87: 962-968.

Recharte, A. 1995. Parásitos de Bufo spinolosus Weigmann, 1835 y Telmatobius marmoratus pseudojleskii Wiegmann, 1834 de la granja K'ayra, Cusco. Biotempo, Lima, 2: 87-90.

Rego, A.A. 1995. A new classification of the cestode Order Proteocephalidea Mola. Revista Brasileira de Zoologia, Curitiba, 12: 791-814.

Rodríguez, L.O.; J.H. CóRdova \& J. ICOChea. 1993. Lista preliminar de los anfibios del Perú. Publicaciones del Museo de Historia Natural, Universidad Nacional Mayor de San Marcos 45: 1-22.

Sarmiento, L.; M. Tantaleán \& A. Huiza. 1999. Nemátodos parásitos del hombre y de los animales en el Perú. Revista Peruana de Parasitologia, Lima, 14: 9-65.

Tantaleán, M. V. \& L. García. 1989. Contribución al estudio de los helmintos parásitos de anfibios del Perú. Boletín de Lima 64: 69-77.

1993. Trematodes de la familia Gorgoderidae en anfibios Leptodactylidae de la región altoandina del Perú. Bol. Lima 85: 25-27.

Tantaleán, M.V.; L. Sarmiento \& A. Huiza. 1992. Digeneos (Trematoda) del Perú. Boletín de Lima 80: 47-84.

Yoder, H.R. \& J.R. Coggins. 1996. Helminth communities in the northern spring peeper, Pseudacris c. crucier Wied, and the wood frog, Rana sylvatica Le Conte from Southeastern Wiscosin. Journal of the Helminthological Society of Washington 63: 211-214.

ZAR, J.H. 1996. Biostatistical Analysis. New Jersey, PrenticeHall, Inc., $3^{\text {th }}$ ed., 662p.

Recebido em 30.IX.2002; aceito em 13.II.2003. 